\title{
Estudio del crecimiento de la estatura en una muestra de niños, niñas y adolescentes sanos de Córdoba, Argentina
}

\author{
Height growth study of healthy children and adolescents from Córdoba, Argentina
}

\author{
María Eloísa Cuestas ${ }^{\mathrm{a}}$, María Elisabeth Cieri ${ }^{\mathrm{b}}$, María de las Mercedes Ruiz Brünner $^{\mathrm{b}}$, Eduardo Cuestas ${ }^{\mathrm{b}, \mathrm{c}}$
}

\begin{abstract}
aDoctora en Física. Especializada en Estadística. Instituto de Física Enrique Gaviola, Consejo Nacional de Investigaciones Científicas y Técnicas. Facultad de Matemáticas, Astronomía y Física, Universidad Nacional de Córdoba. Argentina

bLicenciada en Nutrición. Especialista en Nutrición Infantil. Instituto de Investigaciones en Ciencias de la Salud, Universidad Nacional de Córdoba, Consejo Nacional de Investigaciones Científicas y Técnicas. Argentina

cServicio de Pediatría y Neonatología. Hospital Privado Universitario de Córdoba. Instituto Universitario de Ciencias Biomédicas de Córdoba. Argentina
\end{abstract}

Recibido: 17 de marzo de 2020; Aceptado: 22 de julio de 2020

¿Qué se sabe del tema que trata este estudio?

Ante la carencia de estudios longitudinales es posible obtener la curva media del crecimiento de la estatura en una población a partir de una muestra transversal utilizando el modelo 1 de Preece-Baines.
¿Qué aporta este estudio a lo ya conocido?

El modelo 1 de Preece-Baines permitió estimar satisfactoriamente la edad pico del estirón, la velocidad de crecimiento en dicho punto y la talla final adulta esperada en una muestra de de niños, niñas y adolescentes sanos de Córdoba, Argentina.

\section{Resumen}

Objetivo: Describir el crecimiento en estatura, estimar la edad pico del estirón, la velocidad de crecimiento en dicho punto, la talla final adulta esperada y los patrones diferenciales en una muestra poblacional de ambos sexos. Sujetos y Método: Se realizó un estudio transversal recabando prospectivamente datos demográficos, clínicos y antropométricos en sujetos sanos de ambos sexos; entre 2015 y 2016. Se calcularon los percentiles para la estatura mediante el método LMS (sesgo, mediana y coeficiente de variabilidad) y luego se ajustaron dichos valores utilizando el modelo 1 de PreeceBaines. Resultados: Se evaluaron 861 sujetos, edades 2 - 18 años, 377 varones y 484 mujeres. La edad estimada al pico del estirón $\left(\mathrm{h}_{\theta}\right)$ fue de 13,6 años en los niños y de 11,0 años en las niñas, con una velocidad de crecimiento lineal en ese punto $\left(\mathrm{V}_{2}\right)$ de $6,4 \mathrm{~cm} /$ año para ambos sexos. La estatura adulta media esperada $\left(h_{1}\right)$ se estimó en $173,7 \mathrm{~cm}$ en los chicos y en $160,0 \mathrm{~cm}$ en las chicas. Conclusiones: El modelo 1 de Preece-Baines permitió estimar satisfactoriamente la edad pico del estirón, la velocidad de crecimiento en dicho punto y la talla final adulta esperada.
Palabras clave:

Niños;

Adolescentes;

Crecimiento;

Estatura

Correspondencia:

Eduardo Cuestas

ecuestas@hospitalprivadosa.com.ar 


\section{Abstract}

Objective: Based on a sample of children and adolescents of both genders, our objective is to describe height growth, estimate the peak age at growth spurt, growth rate at this point, the final adult height expected, and differential patterns. Subjects and Method: A cross-sectional study was conducted using demographic, clinical, and anthropometric data collected prospectively from children and adolescents of both sexes between 2015 and 2016. Height percentiles were calculated using the LMS (skewness, median, and coefficient of variation) method and then adjusted using the Preece-Baines model 1. Results: We evaluated 861 participants (484 girls, 377 boys), aged between 2 and 18 years. The estimated peak age at growth spurt $\left(\mathrm{h}_{\theta}\right)$ was 13.6 years in boys and 11.0 years in girls, with a peak growth rate $\left(\mathrm{V}_{2}\right)$ at this point of $6.4 \mathrm{~cm} /$ year for both sexes. The mean expected adult height $\left(\mathrm{h}_{1}\right)$ was $173.7 \mathrm{~cm}$ in boys and $160.0 \mathrm{~cm}$ in girls. Conclusions: Preece-Baines model 1 provides satisfactory estimates for the peak age at growth spurt, peak growth rate at this point, and final expected adult height.

\section{Keywords:}

Children;

Adolescents;

Growth;

Height

\section{Introducción}

Es esencial conocer la estatura de la población de referencia para valorar el crecimiento lineal en niños, niñas y adolescentes ${ }^{1,2}$. Como el crecimiento ocurre simultáneamente con el desarrollo puberal, existe también interés en conocer la edad al inicio y al pico del estirón, y en determinar la magnitud y velocidad del crecimiento que se debe esperar en dichos puntos durante la valoración del crecimiento y desarrollo ${ }^{3}$.

Si bien los estudios longitudinales de crecimiento y desarrollo constituyen el método ideal para describir la magnitud y velocidad del crecimiento ${ }^{4}$, en su defecto, diferentes modelos matemáticos pueden utilizarse para conocer las variaciones del crecimiento estatural de una población mediante funciones específicas en estudios transversales ${ }^{5}$. El modelo de Preece-Baines 1 (PB1) se adapta al estudio del crecimiento de la estatura y ha sido aplicado para describirla en muestras transversales desde la infancia hasta el final de la adolescencia ${ }^{6-8}$. El PB1 incluye parámetros matemáticos y biológicos para determinar la edad de ocurrencia, la magnitud y la velocidad de crecimiento durante las diferentes etapas del desarrollo hasta alcanzar la talla adulta.

Por lo que sabemos, no se ha investigado previamente el crecimiento estatural en una muestra de población argentina contemporánea en estudios longitudinales ni ajustando los datos con el modelo PB1 en estudios transversales.

Los objetivos del presente trabajo fueron entonces:

1) Estimar la edad al inicio y al pico del estirón.

2) Estimar la velocidad de crecimiento al inicio y al pico del estirón.

3) Estimar la estatura al inicio, al pico del estirón y la talla final adulta.

4) Describir los patrones diferenciales entre los sexos.

\section{Sujetos y Método}

Se planeó un estudio de corte transversal. De una población constituida por 30.207 sujetos de 2 a 18 años de edad (15.405 niñas y 14.802 niños) perteneciente a un plan de atención médica integral privado por sistema de prepago, el cual se atiende única y exclusivamente en el Hospital Privado Universitario de Córdoba (Argentina), se escogió al azar 80 sujetos ( 40 niños y 40 niñas) por cada estrato etario de los 2 a los 18 años, invitándose 1.280 sujetos a participar del estudio.

Los datos de la estatura se recabaron prospectivamente. Se incluyeron niños, niñas y adolescentes sanos de 2 y 18 años. Se excluyeron los niños, niñas y adolescentes con peso de nacimiento $<2.500 \mathrm{~g}$, amputaciones, incapacidad para moverse, enfermedades crónicas, trastornos congénitos, genéticos o medicación que pudiera afectar o haber afectado el crecimiento. Las mediciones se realizaron en el Hospital Privado Universitario de Córdoba (Argentina) entre el $1^{\circ}$ de enero de 2015 y el 31 de diciembre de 2016.

Se obtuvo consentimiento informado de los padres o cuidadores y asentimiento informado de los niños, niñas y adolescentes. Este estudio fue aprobado por el Comité Institucional de Ética de nuestro hospital. Los datos antropométricos fueron obtenidos según las guías nacionales, por cuatro investigadores ${ }^{6-9}$. El error de medición se controló mediante prueba de concordancia intra e interobservador como hemos descripto previamente ${ }^{10}$. La estatura se midió utilizando estadiómetros mecánicos marca Seca 216 (Hamburgo, Alemania). Las lecturas se realizaron hasta el último $\mathrm{mm}$ completo. Además, los padres o cuidadores respondieron un cuestionario sobre antecedentes demográficos y médicos.

Los datos continuos normales se probaron con la prueba de Kolmogorov-Smirnov y se informaron como media \pm desviación estándar. Los datos discretos 
se informaron en frecuencias absolutas y relativas con intervalos de confianza del 95\%.

Desarrollamos curvas de percentiles para estatura por sexo suavizadas mediante el método $\mathrm{LMS}^{11}$ utilizando el paquete GAMLSS (Generalized Additive Models for Location Scale and Shape) para R. El método utiliza una probabilidad máxima semi-paramétrica para estimar curvas de crecimiento suavizadas que pueden resumirse mediante la mediana $(M)$, el coeficiente de variación generalizado (S) y la potencia de Box-Cox para el sesgo (L) al mismo tiempo que representan la curtosis $(\mathrm{T})^{12}$ Se aplicaron splines (un spline es una curva diferenciable definida en porciones mediante polinomios) ponderados localmente para ajustar las curvas según edad y obtener así funciones de ajuste objetivo que fueron utilizadas para calcular los percentiles 3, 50 y 97 .

La validación externa se realizó comparando los valores generados de los datos de nuestra con los valores de referencia nacionales para niños y niñas de 2 a 18 años 9 .

Según Rosique Gracia y colaboradores el modelo PB1se adapta al estudio del crecimiento en el período de la adolescencia y se ha utilizado para describir el crecimiento estatural medio de muestras longitudinales y transversales. La curva de distancia que describe el PB1 queda resumida por cinco parámetros: h1, s0, s1 y. El parámetro h1 representa la asíntota superior (talla adulta) si se modelizan casos, o la talla adulta en el percentil 50 si se modelizan muestras. Es el único parámetro con interpretación biológica, ya que el resto son parámetros de forma y sólo se usan para construir la función. De forma aproximada; se relaciona con la talla en la pendiente decreciente del pico del estirón, los parámetros s0 y s1 se relacionan con los incrementos medios durante el estirón (describen la forma del pico) y el parámetro se relaciona con la edad de. Sin embargo, la primera derivada del modelo es útil para obtener algunos parámetros de interés, como la edad y la talla al inicio del estirón y en el pico del estirón. La aplicación del PB1 a datos transversales produce curvas percentilares muy similares a las derivadas de muestreos longitudinales, salvo en la descripción de la variancia en la adolescencia y en el efecto esperado de aplanamiento de la curva de velocidad. Por ello, es más correcto hablar de "curva de seudovelocidad" en los estudios transversales, siendo la talla adulta y la edad del pico del estirón los parámetros poblacionales que se derivan con menor sesgo respecto a los estudios longitudinales ${ }^{8}$.

Se utilizó el modelo de PB1 para estimar los siguientes parámetros matemáticos: (estatura adulta en $\mathrm{cm}$ ), (estatura a la edad en $\mathrm{cm}$ ), (constantes de incrementos medios, pre-puberal y puberal en $\mathrm{cm} / \mathrm{año}$ ) y (edad en la pendiente decreciente del pico del estirón en $\mathrm{cm}$ ). Los parámetros fueron estimados en base a mínimos cuadrados. Los valores correspondientes fueron generados mediante la aplicación del modelo PB1 de la siguiente manera:

$$
y=h_{1}-\frac{2\left(h_{1}-h_{\theta}\right)}{e^{s_{\theta}(t-\theta)}+e^{s_{1}(t-\theta)}}
$$

La primera derivada del modelo (fue utilizada para establecer los siguientes parámetros biológicos: (edad al inicio del estirón en años), (velocidad al inicio del estirón en cm/año), (edad al pico del estirón, en años) y (velocidad al pico del estirón en cm/año) ${ }^{6}$.

Los datos se analizaron con el programa $\mathrm{R}$ versión 3.5.1.

\section{Resultados}

De los 1.280 sujetos invitados para el estudio, 1.025 concurrieron para elegibilidad. Luego de la evaluación, 164 fueron excluidos. La muestra quedó conformada por 861 sujetos, 484 niñas $(56,2 \%$ IC $95 \% 52,8$ a 59,5$)$ con una estatura media de $138,1 \pm 20,3 \mathrm{~cm}$ y 377 niños $(43,8 \%$ IC $95 \% 40,4$ a 47,2$)$ con una estatura media de $138,3 \pm 24,5 \mathrm{~cm}$. La muestra se dividió en 16 estratos de 1 año de los 2 a los 18 años para cada sexo. El rango de edades decimales para las niñas fue de 2.375 a 18.923 años y para los niños de 2.013 a 18.948 años.

Los percentiles calculados para la altura con el uso de los parámetros LMS específicos por edad y sexo que generamos a partir de nuestros datos se compararon con los percentiles 3,50 y 97 de la referencia nacional para niños y niñas de 2 a 18 años. Las diferencias se calcularon tomando como minuendo el valor de referencia nacional ${ }^{6}$. Para ambos sexos los datos se ajustaron razonablemente con una diferencia relativa media menor al $1 \%$. Ambas curvas presentan prácticamente la misma talla a los 18 años en ambos sexos (tablas 1 y 2).

En la figura 1 se muestran las curvas de distancia de la estatura con los datos observados en nuestra muestra y los estimados por el modelo de PB1 para niños y niñas. Con una estatura adulta estimada $\left(h_{1}\right)$ de 173,7 y $160,0 \mathrm{~cm}$, una estatura media estimada al pico del estirón (de 160,6 y 146,0 cm y una edad estimada del pico del estirón $(\theta)$ de 13,6 y 11,0 años para los niños y niñas respectivamente.

Las curvas de velocidad de la estatura para los datos observados en la muestra y los estimados por el modelo de PB1 para niños y niñas se muestran en la figura 2. Con una edad al inicio del estirón $\left(T_{1}\right)$ de 8,9 y 6,4 años, una edad al inicio del pico del estirón $\left(\mathrm{T}_{2}\right)$ de 12,6 y 10,6 años y una velocidad de estatura al inicio del estirón $\left(\mathrm{V}_{1}\right)$ de 5,25 y $5,28 \mathrm{~cm} /$ año para niños y niñas 


\begin{tabular}{|c|c|c|c|c|c|c|c|c|c|c|c|c|c|c|}
\hline Niños & Edad & $\mathrm{N}$ & $\begin{array}{c}\text { CBA } \\
\text { p3 }\end{array}$ & $\begin{array}{l}\text { CBA } \\
\text { p50 }\end{array}$ & $\begin{array}{l}\text { CBA } \\
\text { p97 }\end{array}$ & SAP p3 & $\begin{array}{l}\text { SAP } \\
\text { p50 }\end{array}$ & $\begin{array}{l}\text { SAP } \\
\text { p97 }\end{array}$ & $\begin{array}{c}\Delta \mathrm{p} 3 \\
\mathrm{~cm}\end{array}$ & $\begin{array}{c}\Delta p 3 \\
\%\end{array}$ & $\begin{array}{c}\Delta \mathrm{p} 50 \\
\mathrm{~cm}\end{array}$ & $\begin{array}{c}\Delta \text { p50 } \\
\%\end{array}$ & $\begin{array}{c}\Delta \mathrm{p} 97 \\
\mathrm{~cm}\end{array}$ & $\begin{array}{c}\Delta p 97 \\
\%\end{array}$ \\
\hline & 2 & 26 & 79,7 & 86,6 & 93,6 & 82,0 & 87,8 & 93,5 & 2,4 & 1,3 & 1,2 & 1,2 & 0,0 & 1,1 \\
\hline & 3 & 24 & 87,3 & 95,0 & 102,7 & 89,3 & 96,4 & 103,5 & 2,0 & 1,1 & 1,4 & 1,1 & 0,9 & 1,0 \\
\hline & 4 & 25 & 94,5 & 102,9 & 111,2 & 94,6 & 102,6 & 110,6 & 0,0 & 1,1 & $-0,3$ & 1,0 & $-0,6$ & 0,9 \\
\hline & 5 & 22 & 101,0 & 110,0 & 118,9 & 99,5 & 107,9 & 116,4 & $-1,6$ & 1,0 & $-2,0$ & 0,9 & $-2,5$ & 0,8 \\
\hline & 6 & 25 & 106,8 & 116,3 & 125,8 & 105,2 & 114,2 & 123,1 & $-1,6$ & 0,9 & $-2,2$ & 0,9 & $-2,7$ & 0,8 \\
\hline & 7 & 21 & 112,2 & 122,1 & 132,1 & 110,7 & 120,2 & 129,7 & $-1,4$ & 0,9 & $-1,9$ & 0,8 & $-2,4$ & 0,8 \\
\hline & 8 & 22 & 117,4 & 127,8 & 138,2 & 115,8 & 125,9 & 136,1 & $-1,6$ & 0,9 & $-1,9$ & 0,8 & $-2,2$ & 0,7 \\
\hline & 9 & 20 & 122,2 & 133,0 & 143,8 & 120,2 & 131,1 & 141,9 & $-2,0$ & 0,8 & $-1,9$ & 0,8 & $-1,9$ & 0,7 \\
\hline & 10 & 21 & 126,9 & 138,0 & 149,2 & 124,0 & 135,8 & 147,5 & $-2,8$ & 0,8 & $-2,3$ & 0,7 & $-1,7$ & 0,7 \\
\hline & 11 & 20 & 132,2 & 143,7 & 155,2 & 127,5 & 140,3 & 153,0 & $-4,7$ & 0,8 & $-3,4$ & 0,7 & $-2,2$ & 0,6 \\
\hline & 12 & 22 & 138,2 & 150,1 & 161,9 & 131,3 & 145,4 & 159,4 & $-7,0$ & 0,7 & $-4,7$ & 0,7 & $-2,5$ & 0,6 \\
\hline & 13 & 20 & 144,4 & 156,6 & 168,8 & 136,1 & 151,5 & 167,0 & $-8,3$ & 0,7 & $-5,1$ & 0,6 & $-1,8$ & 0,6 \\
\hline & 14 & 21 & 150,0 & 162,4 & 174,8 & 142,5 & 158,4 & 174,3 & $-7,5$ & 0,7 & $-4,0$ & 0,6 & $-0,5$ & 0,6 \\
\hline & 15 & 22 & 154,6 & 167,1 & 179,6 & 149,2 & 164,6 & 180,0 & $-5,4$ & 0,6 & $-2,5$ & 0,6 & 0,4 & 0,6 \\
\hline & 16 & 23 & 157,9 & 170,3 & 182,8 & 154,6 & 169,1 & 183,6 & $-3,2$ & 0,6 & $-1,2$ & 0,6 & 0,8 & 0,5 \\
\hline & 17 & 21 & 159,7 & 172,0 & 184,3 & 158,1 & 171,7 & 185,3 & $-1,6$ & 0,6 & $-0,3$ & 0,6 & 1,0 & 0,5 \\
\hline & 18 & 22 & 160,9 & 173,0 & 185,1 & 159,7 & 172,7 & 185,7 & $-1,2$ & 0,6 & $-0,2$ & 0,6 & 0,7 & 0,5 \\
\hline
\end{tabular}

Tabla 2. Diferencia $(\Delta)$ absoluta $(\mathrm{cm})$ y relativa (\%) de los percentiles $(\mathrm{p}) 3,50$ y 97 de estatura entre nuestra muestra (CBA) y la referencia nacional (SAP)

\begin{tabular}{|c|c|c|c|c|c|c|c|c|c|c|c|c|c|c|}
\hline Niñas & Edad & $n$ & $\begin{array}{l}\text { CBA } \\
\text { p3 }\end{array}$ & $\begin{array}{l}\text { CBA } \\
\text { p50 }\end{array}$ & $\begin{array}{l}\text { CBA } \\
\text { p97 }\end{array}$ & SAP p3 & $\begin{array}{l}\text { SAP } \\
\text { p50 }\end{array}$ & $\begin{array}{l}\text { SAP } \\
\text { p97 }\end{array}$ & $\begin{array}{c}\Delta \mathrm{p3} \\
\mathrm{cm}\end{array}$ & $\begin{array}{c}\Delta p 3 \\
\%\end{array}$ & $\begin{array}{c}\Delta \mathrm{p} 50 \\
\mathrm{~cm}\end{array}$ & $\begin{array}{c}\Delta \text { p50 } \\
\%\end{array}$ & $\begin{array}{c}\Delta \mathrm{p} 97 \\
\mathrm{~cm}\end{array}$ & $\begin{array}{c}\Delta p 97 \\
\%\end{array}$ \\
\hline & 2 & 30 & 79,1 & 86,1 & 93,2 & 80,3 & 86,4 & 92,4 & 1,2 & 1,3 & 0,2 & 1,2 & $-0,8$ & 1,1 \\
\hline & 3 & 31 & 86,0 & 93,6 & 101,3 & 87,8 & 95,0 & 102,3 & 1,8 & 1,2 & 1,4 & 1,1 & 1,0 & 1,0 \\
\hline & 4 & 28 & 92,8 & 101,0 & 109,3 & 92,9 & 101,2 & 109,5 & 0,2 & 1,1 & 0,2 & 1,0 & 0,2 & 0,9 \\
\hline & 5 & 29 & 99,2 & 108,0 & 116,9 & 97,7 & 106,7 & 115,7 & $-1,5$ & 1,0 & $-1,3$ & 0,9 & $-1,2$ & 0,9 \\
\hline & 6 & 31 & 105,3 & 114,7 & 124,0 & 103,4 & 113,0 & 122,6 & $-2,0$ & 0,9 & $-1,7$ & 0,9 & $-1,5$ & 0,8 \\
\hline & 7 & 29 & 111,2 & 121,1 & 130,9 & 108,6 & 118,8 & 129,0 & $-2,6$ & 0,9 & $-2,3$ & 0,8 & $-2,0$ & 0,8 \\
\hline & 8 & 27 & 117,0 & 127,4 & 137,7 & 113,2 & 124,1 & 135,0 & $-3,8$ & 0,9 & $-3,3$ & 0,8 & $-2,7$ & 0,7 \\
\hline & 9 & 28 & 122,9 & 133,7 & 144,6 & 117,3 & 129,2 & 141,1 & $-5,6$ & 0,8 & $-4,5$ & 0,7 & $-3,5$ & 0,7 \\
\hline & 10 & 29 & 128,7 & 140,0 & 151,3 & 121,4 & 134,6 & 147,7 & $-7,3$ & 0,8 & $-5,4$ & 0,7 & $-3,6$ & 0,7 \\
\hline & 11 & 30 & 134,0 & 145,7 & 157,4 & 126,1 & 140,6 & 155,0 & $-7,9$ & 0,7 & $-5,2$ & 0,7 & $-2,4$ & 0,6 \\
\hline & 12 & 29 & 138,5 & 150,5 & 162,5 & 132,1 & 147,0 & 161,9 & $-6,4$ & 0,7 & $-3,5$ & 0,7 & $-0,5$ & 0,6 \\
\hline & 13 & 28 & 142,1 & 154,2 & 166,3 & 138,8 & 152,9 & 167,0 & $-3,2$ & 0,7 & $-1,3$ & 0,6 & 0,7 & 0,6 \\
\hline & 14 & 27 & 144,6 & 156,8 & 169,0 & 144,3 & 157,2 & 170,0 & $-0,3$ & 0,7 & 0,4 & 0,6 & 1,0 & 0,6 \\
\hline & 15 & 28 & 146,1 & 158,3 & 170,5 & 147,6 & 159,6 & 171,6 & 1,4 & 0,7 & 1,3 & 0,6 & 1,1 & 0,6 \\
\hline & 16 & 29 & 147,0 & 159,0 & 171,1 & 149,0 & 160,5 & 172,1 & 2,0 & 0,7 & 1,5 & 0,6 & 1,0 & 0,6 \\
\hline & 17 & 27 & 147,4 & 159,4 & 171,3 & 149,3 & 160,7 & 172,2 & 1,9 & 0,7 & 1,4 & 0,6 & 0,9 & 0,6 \\
\hline & 18 & 24 & 147,8 & 159,6 & 171,4 & 149,3 & 160,7 & 172,2 & 1,5 & 0,7 & 1,1 & 0,6 & 0,8 & 0,6 \\
\hline
\end{tabular}




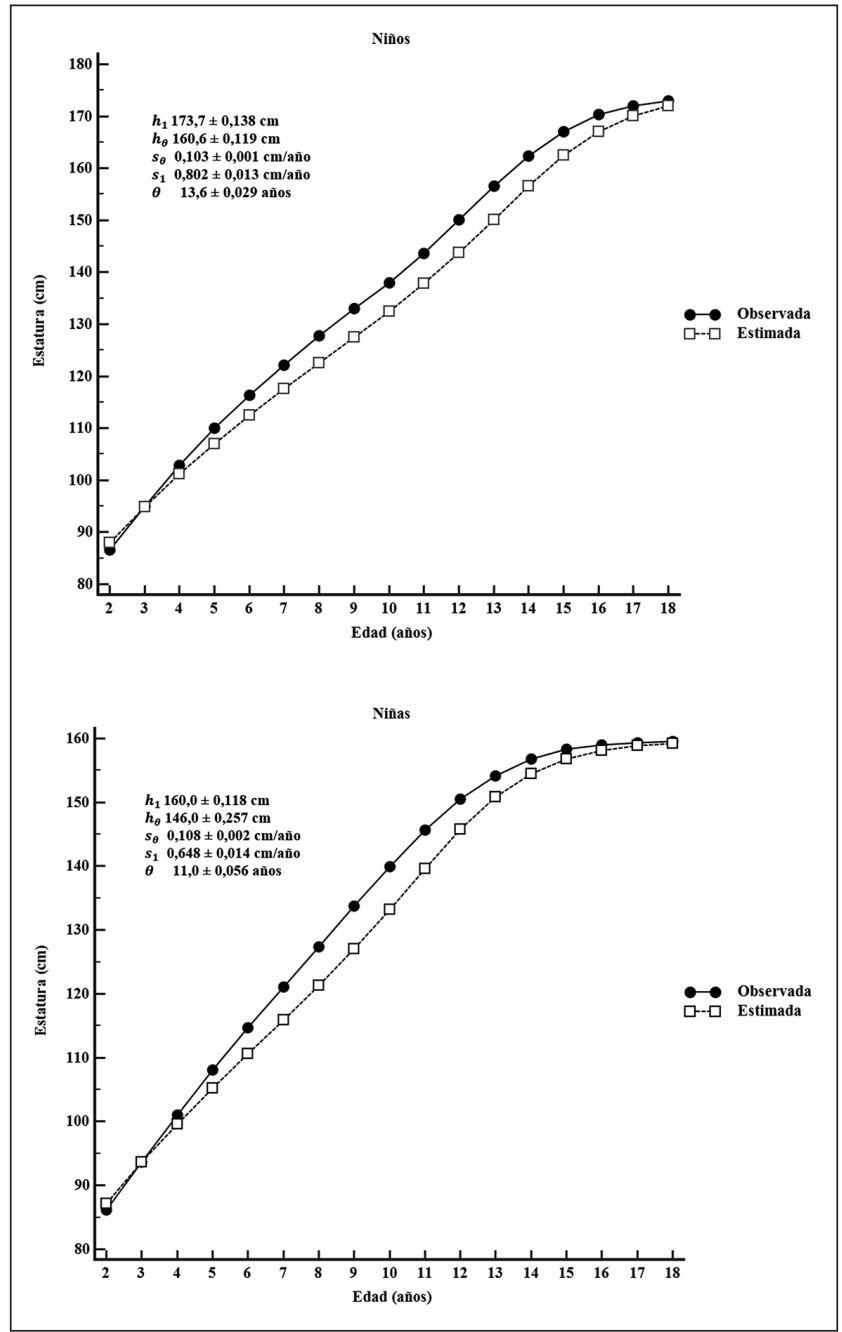

Figura 1. Curvas de distancia para la estatura con los datos observados en nuestra muestra (Observada) y los estimados por el modelo de Preece-Baines 1 (Estimada) para niños (arriba) y niñas (abajo). La dispersión se expresa en \pm EEE (error estándar de la estimación). Significado de $h_{1}=$ talla final media, $h_{\theta}=$ estatura media al pico del estirón y $\theta=$ edad al pico del estirón.

respectivamente y de $6,40 \mathrm{~cm} /$ año en los niños y de $6,44 \mathrm{~cm} /$ año en las niñas al pico del estirón $\left(\mathrm{V}_{2}\right)$ ".

Las diferencias que encontramos entre los niños y las niñas en los parámetros matemáticos y biológicos se describen en la figura 3. Los niños respecto a las niñas presentaron una estatura final 8,6\% mayor, una edad al pico del estirón 2 años mayor y una velocidad media de crecimiento ligeramente menor tanto prepuberal como puberal.

\section{Discusión}

Se presenta por primera vez una referencia para valorar la edad de inicio y de pico del estirón junto a la magnitud y

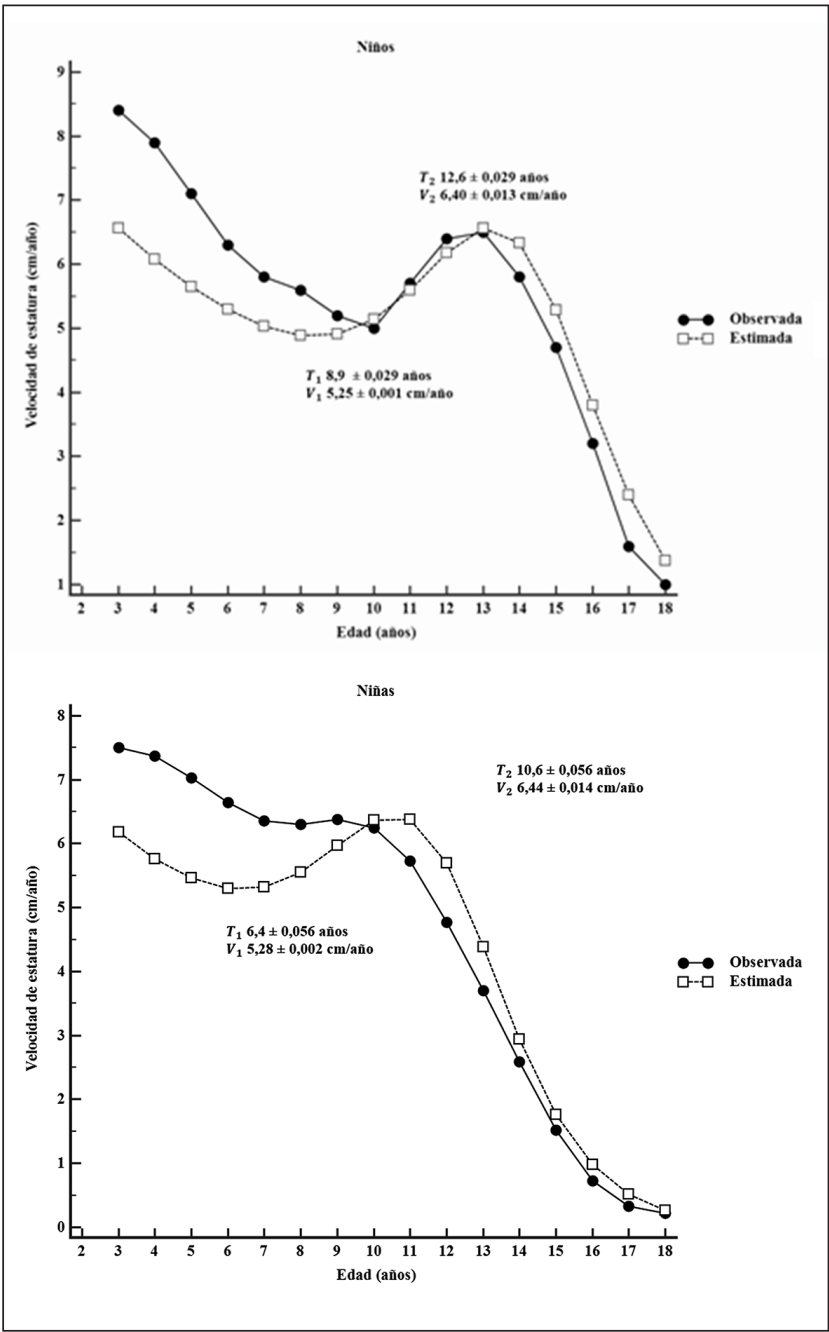

Figura 2. Curvas de velocidad para la estatura con los datos observados en nuestra muestra (Observada) y los estimados por el modelo de Preece-Baines 1 (Estimada) para niños (arriba) y niñas (abajo). La dispersión se expresa en \pm EEE (error estándar de la estimación). Significado de T1 = edad al inicio del estirón, T2 = edad al pico del estirón, V1 = velocidad de crecimiento al inicio del estirón y V2 = velocidad de crecimiento al pico del estirón.

velocidad de crecimiento esperada en dichos puntos usando datos derivados de una muestra trasversal de una población cordobesa contemporánea constituida por niños, niñas y adolescentes sanos, cuya curva se ajusta muy bien al estándar nacional ${ }^{9}$ utilizando el modelo PB1.

Comparando nuestros resultados con el único estudio trasversal existente modelado con PB1 en una población similar $^{8}$, encontramos que la talla final (h1) es menor en nuestra muestra (173,7 cm vs 176,2 para niños y 160,0 vs 163,5 para las niñas), de acuerdo con la variación inter-poblacional esperada ${ }^{13}$. Mientras que la edad al pico del estirón () es muy similar en ambas poblaciones tanto en los niños (13,6 vs 13,7) como en las niñas $(11,04$ vs 11,02).

Comparada con los datos de la referencia nacional vi- 


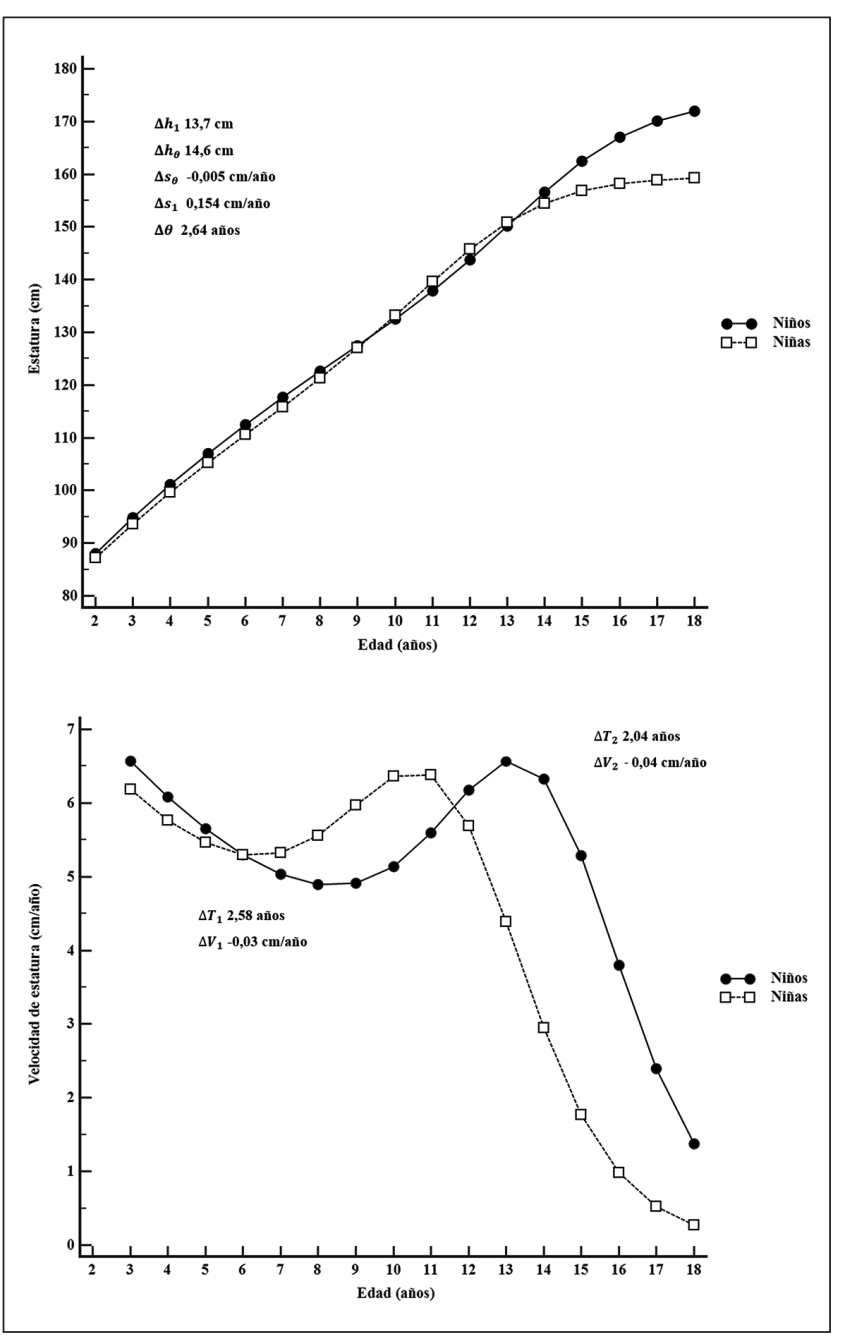

Figura 3. Curvas de diferencias $(\Delta)$ de los parámetros matemáticos: $h_{1}$ (estatura adulta), $h_{\theta}$ (estatura a la edad del pico del estirón), $s_{1}$ y $s_{\theta}$ (constantes de incrementos medios, puberal y prepuberal) y $\theta$ (edad en la pendiente decreciente del pico del estirón) (arriba) y los parámetros biológicos: $T_{1}$ (edad al inicio del estirón), $V_{1}$ (velocidad al inicio del estirón), $T_{2}$ (edad al pico del estirón) y $V_{2}$ (velocidad al pico del estirón) entre niños y niñas.

gente de hace cinco décadas ${ }^{9}$ la talla a los 18 años observada en nuestra muestra fue $1 \mathrm{~cm}$ mayor en los varones y $0,7 \mathrm{~cm}$ menor en las mujeres. Estos resultados están en concordancia con la evidencia disponible de que la talla no se ha modificado en Argentina en las últimas décadas ${ }^{14,15}$.

Comparados con datos de desarrollo puberal de niños y niñas argentinos publicados en $1976^{16}$, los sujetos de este estudio presentaron una edad al inicio del estirón $\left(\mathrm{T}_{1}\right)$, correlativa al inicio puberal, 2,2 años menor en los niños $(8,9$ $v s 11,1)$ y 4,4 años menor en las niñas $(6,4 v s 10,8)$, hecho que probablemente obedezca a la tendencia de inicio puberal más temprano que se observa también en otros países ${ }^{17}$.

La merma observada en la talla final de las mujeres también puede estar relacionada con la edad más temprana de la menarca ${ }^{18}$, pero esta observación debe ser tomada con cautela, hasta que se confirme o refute mediante un estudio longitudinal prospectivo que investigue específicamente la influencia de la edad de la menarca sobre la talla final adulta en nuestro medio. El crecimiento ocurrió a tasas similares en ambos sexos, pero durante más tiempo en los niños, explicando su mayor estatura final. En los niños estadounidenses la velocidad pico del estirón es de $9.5 \mathrm{~cm} /$ año, mientras que en las niñas es de $8,3 \mathrm{~cm} / \mathrm{año}^{19}$.

Las diferentes velocidades al pico del estirón $\left(V_{2}\right)$ entre niños y niñas han sido atribuidas al mayor crecimiento óseo dependiente de la testosterona que ocurre en los varones durante la pubertad ${ }^{20}$. Lamentablemente, no existen datos longitudinales de niños argentinos que nos permitan realizar una comparación al respecto, sin embargo, los resultados de nuestro estudio transversal, muestran una velocidad al pico del estirón $\left(\mathrm{V}_{2}\right)$ bastante más baja que las reportadas en el estudio longitudinal estadounidense ${ }^{19} \mathrm{y}$ sin diferencia aparente entre niños y niñas, aunque es muy similar a las informadas por el estudio transversal español que utilizó el modelo de PB1 $(6,1 \mathrm{~cm} /$ año para los niños y 5,8 cm/año para las niñas $)^{8}$. Muy posiblemente el modelo de PB1 incurre en una estimación más baja de la velocidad al pico del estirón comparado con los modelos utilizados en estudios longitudinales, por el efecto de aplanamiento de la curva de velocidad, aunque los valores se encuentran dentro de los rangos de 6,1 a 12,3 cm/año para niños y de 6,2 a 10,3 cm/ año para niñas establecidos por Tanner y colaboradores ${ }^{21,22}$.

Respecto a las diferencias en la velocidad al pico del estirón entre niños y niñas, existe actualmente un creciente número de investigaciones realizadas en gemelos, que demuestran que ambos sexos crecen a la misma velocidad, si esta se ajusta a la maduración puberal, y no a la edad, adjudicando la mayor estatura final en los varones a la mayor duración del crecimiento tanto prepuberal como intrapuberal y no a una mayor velocidad de crecimiento, este hecho es probablemente mejor reflejado por el modelo de PB1 que por los modelos utilizados en los estudios longitudinales ${ }^{23-25}$.

Este trabajo se encuentra limitado principalmente por su carácter transversal, por lo que debe considerarse como una estimación provisoria hasta contar con datos definitivos generados en estudios longitudinales. Sin pretender substituir la necesidad de contar con datos nacionales longitudinales, los parámetros presentados aquí provienen, sin embargo, de un análisis prolijo que ha proporcionado ajustes aceptables a juzgar por los valores de los errores estándar estimados con los observados y que ha controlado adecuadamente los sesgos potenciales para determinar la edad y velocidad al inicio y al pico del estirón, pero que puede haber subestimado la edad al inicio del estirón, equivalente a la edad de mínima velocidad de la estatura en los estudios longitudinales.

La diferencia de 2,5 años para la edad del inicio del estirón entre niñas y niños que encontramos en este trabajo se encuentra dentro de los rangos esperados. Creemos haber solucionado este inconveniente incluyendo niños y niñas de 
todos los rangos etarios desde los 2 hasta los 18 años con tamaños muestrales representativos en cada estrato como plantean Zemel y Johnston en su trabajo sobre validación e interpretación del modelo de PB1. Estos autores plantean que el método PB1 se puede aplicar con éxito a datos de crecimiento de corte transversal para hacer inferencias tanto sobre el momento del pico de crecimiento acelerado como de la duración de este período de crecimiento en el adolescente. Los datos para cada muestra de corte transversal se dividieron por sexo y la estatura media a cada edad se calculó y analizó utilizando el modelo de PB1. De esta manera, las inferencias sobre los procesos de crecimiento longitudinal, como el momento del pico de crecimiento de los adolescentes, tomadas de datos transversales, se pudieron probar con registros verdaderamente longitudinales. Las comparaciones realizadas por estos autores, entre las muestras transversales seleccionadas al azar y los registros longitudinales para los mismos niños revelaron algunas diferencias entre los dos enfoques. Al utilizar el modelo de PB1, debe tenerse en cuenta que la curva de velocidad promedio del modelo matemático es más amplia y plana que la curva de cualquier individuo aislado, por lo que debe utilizarse sólo para realizar inferencias poblacionales y no individuales.

La velocidad al pico del estirón fue menor de la determinada longitudinalmente, pero la mayoría de los otros parámetros examinados, en particular la edad en el pico del estirón, coincide con los datos de datos de sección transversal, aunque la edad de inicio del estirón, especialmente en las niñas es significativamente menor que la determinada por datos longitudinales en consecuencia con los hallazgos de nuestro estudio, lo que constituye una limitación relevante del método. Otro elemento importante a tener en cuenta es cuando esta técnica se utiliza para comparar poblaciones, la variabilidad que ocurre dentro de una población y entre las muestras seleccionadas de la misma población debe tenerse en cuenta antes de llegar a conclusiones definitivas $^{26}$.
En conclusión, el modelo de PB1 permitió estimar satisfactoriamente la edad al inicio y al pico del estirón, la velocidad de crecimiento en dichos puntos y la talla final adulta esperada en nuestra muestra, aunque estos datos deben considerarse provisorios hasta confirmarlos con datos definitivos originados en estudios longitudinales.

\section{Responsabilidades Éticas}

Protección de personas y animales: Los autores declaran que los procedimientos seguidos se conformaron a las normas éticas del comité de experimentación humana responsable y de acuerdo con la Asociación Médica Mundial y la Declaración de Helsinki.

Confidencialidad de los datos: Los autores declaran que han seguido los protocolos de su centro de trabajo sobre la publicación de datos de pacientes.

\section{Derecho a la privacidad y consentimiento informa-} do: Los autores han obtenido el consentimiento informado de los pacientes y/o sujetos referidos en el artículo. Este documento obra en poder del autor de correspondencia.

\section{Financiamiento}

Este trabajo fue financiado por la Universidad Nacional de Córdoba (Res. SeCyT-UNC 313/16).

\section{Conflicto de intereses}

Los autores declaran no tener conflicto de intereses.

\section{Referencias}

1. Beas F, Contreras I, Maccioni A, Lacassie I, Monckeberg F, Muzzo S. Diagnóstico del retardo del crecimiento en el niño. Rev Chil Pediatr 1968;8:649-53.

2. Muzzo BS. Crecimiento normal y patológico del niño y del adolescente. Rev Chil Nutr 2003;30:92-100.

3. Granados A, Gebremariam A, Lee JM. Relationship Between Timing of Peak Height Velocity and Pubertal Staging in Boys and Girls. J Clin Res Pediatr Endocrinol 2015;7:235 7.
4. Karlberg J, Kwan CW, Gelander L, Albertsson-Wikland K. Pubertal growth assessment. Horm Res 2003;27-35.

5. Simpkin AJ, Sayers A, Gilthorpe MS, Heron J, Tilling K. Modelling height in adolescence: a comparison of methods for estimating the age at peak height velocity. Ann Hum Biol 2017;44(8):715-22.

6. Preece MA, Baines MJ. A new family of mathematical models describing the human growth curve. Ann Hum Biol 1978;5:1-24.

7. Hauspie R, Roelants M. Adolescent Growth. In: Cameron N, Bogin B, eds.
Human Growth and Development. London, Elsevier; 2012:57-79.

8. Rosique Gracia J, Gordón Ramos P, Rebato Ochoa E, et al. Estudio auxológico de muestras contemporáneas e históricas de la población madrileña: aplicación del modelo 1 de Preece-Baines. An Esp Pediatr 2001;54:468-76.

9. Guía para la evaluación del crecimiento físico. Sociedad Argentina de Pediatría. Buenos Aires. 2013.

10. Ruiz Brünner MM, Butler C, Cuestas E. Development of regression equations for estimating height and weight using 
segments in argentine children. Nutrition 2018;57:122-6.

11. Cole TJ. Using LMS method to measure skewness in the NCHS and Dutch National height standards. Ann Hum Biol 1989;16:407-19.

12. Stasinopoulos MD, Rigby RA. Generalized Additive Models for Location Scale and Shape (GAMLSS) in R. J Stat Softw 2007;23(7):1-46.

13. Haas JD, Campirano F. Interpopulation variation in height among children 7 to 18 years of age. Food Nutr Bull 2006;27:S21223.

14. Guimarey LM, Castro LE, Torres MF, et al. Secular changes in body size and body composition in schoolchildren from La Plata City (Argentina). Anthropol Anz 2014;71:287-301.

15. Orden AB, Bucci PJ, Petrone S. Trends in weight, height, BMI and obesity in schoolchildren from Santa Rosa (Argentina), 1990-2005/07. Ann Hum Biol
2013;40:348-54.

16. Lejarraga H, Cusminsky M, Castro EP. Age of onset of puberty in urban Argentinian children. Ann Hum Biol 1976;3:379-81.

17. Karlberg J. Secular trends in pubertal development. Horm Res. 2002;57 S2:19-30.

18. Onland-Moret NC, Peeters PH, van Gils $\mathrm{CH}$, et al. Age at menarche in relation to adult height: the EPIC study. Am J Epidemiol. 2005;162:623-32.

19. Bordini B, Rosenfield RL. Normal pubertal development: part II: clinical aspects of puberty. Pediatr Rev. 2011;32:281-92.

20. Buckler JM, Wild J. Longitudinal study of height and weight at adolescence. Arch Dis Child. 1987;62:1224-32.

21. Tanner JM, Whitehouse RH, Takaishi M. Standards from birth to maturity for height, weight, height velocity, and weight velocity: British children, 1965. I. Arch Dis Child. 1966;41:454-71.

22. Tanner JM, Whitehouse RH, Takaishi M. Standards from birth to maturity for height, weight, height velocity, and weight velocity: British children, 1965. II. Arch Dis Child. 1966;41:613-35.

23. Iuliano-Burns S, Hopper J, Seeman E. The age of puberty determines sexual dimorphism in bone structure: a male/ female co-twin control study. J Clin Endocrinol Metab. 2009;94:1638-43.

24. Almeida M, Laurent MR, Dubois V, Claessens F, O'Brien CA, Bouillon R, Vanderschueren D, Manolagas SC. Estrogens and Androgens in Skeletal Physiologyand Pathophysiology. Physiol Rev. 2017;97:135-87.

25. Gasser T, Kneip A, Binding A, Prader A, Molinari L. The dynamics of linear growth in distance, velocity and acceleration. Ann Hum Biol 1991;18:187-205.

26. Zemel BS, Johnston FE. Application of the Preece-Baines growth model to crosssectional data: Problems of validity and interpretation. Am J Hum Biol 1994;6:56357. 